\title{
Thinking and Research of Application Innovation of Fashion Display Design

\author{
Yi Fang
}

Jilin Engineering Normal University, Changchun City, Jilin Province, China, 130000

434294631@qq.com

Keywords: Clothes; Display design; Technical application; Features

\begin{abstract}
With the continuous improvement of people's living standard in recent decades, people have more requirements for clothes. Fashion display design is a direct platform of consumers and clothes. An excellent fashion display design can promote the marketing of clothing products to directly show the charm of clothes to the consumers. People's aesthetic ideas and minds are also changing because of the rapid changes of times and the continuous integration of various factors, under this background, an excellent fashion display design should be carried out by fully considering and innovating the technical application of fashion display design used for showing the product features with products and the requirements of consumers as the starting point. Only such fashion display design can meet the requirements of market development and the consumers and be in an invincible position in the competition.
\end{abstract}

\section{Introduction}

With the continuous improvement of people's living standard, people have higher and higher requirements on the clothes. As the platform for direct communication of clothing products and consumers, fashion display design is put forward higher requirements. So to speak, an excellent fashion display design not only can promote the marketing of clothing products but also can show and deliver the culture connotation of clothing enterprises to consumers by different product models to highlight the current popular tendency and guide the aesthetic tendency of consumers. However, fashion display design should be fully applied and innovated in clothing marketing system, that is the only way clothing enterprises can be constantly enhanced and improved in the changing market, and occupy and stabilize a seat in the fierce market competition.

\section{Research on Background of Fashion Display Design}

The communication between China and the world becomes more frequent since China affiliated the WTO. Some western artistic thoughts and theories also begin to be integrated into the popular tendency of China which greatly affect the consumption area of China. So to speak, some conceptual consumption ideas have great influence on the Chinese clothing market. Also because of China's entry into WTO, China see the world, China's clothing industry finds the gap and begins to close the gap by taking steps.

In the early times of the development of China's clothing industry, more attention was not paid to fashion display design by the relevant enterprises and people. At that time, it was just considered for clothing industry that the means of improving product competitiveness are obtained by clothes own reform and the advancement of manufacture craft. But with the development of market, people began to notice the effect of fashion display and applied fashion display design to some larger fashion stores. Especially, the diversified fashion display design was integrated into some high-grade fashion cabinets. This situation has been widely accepted by many enterprises, at the same time, the corporate culture is also integrated into for better effect in marketing. Fashion display design can bring direct visual impact and internal psychological reactions to consumers. It will greatly promote the development of clothing enterprises and the guidance of people's aesthetic ideas to use fashion display design well. But for the actual status, although fashion display design 
has been widely applied in our country, there are still some problems in application of fashion display design such as the single operating means, rough style and the lack of design innovation.

\section{Significance of Fashion Display Design}

From the above background analysis, it can be seen that fashion display design is very important for the marketing of clothing enterprises in the development of clothing industry despite of the short history of fashion display design. At the same time, fashion display design can promote the effective connection and communication between enterprises and consumers. In a word, the significance of fashion display design are mainly expressed at the following aspects.

Firstly, fashion design can help to better show product image. Fashion display design is the most direct show of product which has very important influence on the corporate image, design concept, product orientation and other aspects. Fashion display design can directly show the whole style of product and affect the marketing of product. The direct expression of fashion designing conception is carried out by fashion display design, this expression is adopted in the marketing of many premium brands abroad which can directly affect the marketing of product and the transmission of product culture. However, adequate attention should be gave to fashion display design, and the level of fashion display design should be improved to make its full use.

Secondly, display design is conductive to the information communication of businesses and consumers. Eventually, the product needs to be sold, the objects the product face are consumers. However, it needs the help of fashion display design to reduce the distance of products and consumers. In fashion display design, some specific scenes and atmospheres are often created to make the consumers to arouse association related to the life and buying desire. The display design with different style can bring different feelings and visual enjoyment to consumers and meet the different requirements of different consumers, at the same time, consumers can choose their favorite products according to their own requirements, so the close connection between consumers and products is established. At the same time, businesses can make innovation in fashion display design to form a good interaction.

\section{Features Expression of Fashion Display Design}

From the above elaboration, we can know that an excellent fashion display design can not only promote the marketing of clothing products but also show and deliver the cultural connotation of clothing enterprises to the consumers by different product styles to highlight the current popular tendency and guide the aesthetic tendency of consumers.

\section{Fashion Display Design Is the Show of Clothing Product Image}

The most direct show of product is carried out with the help of fashion display design. Under the background of fierce market competition, clothing enterprises transmit their corporate image, clothing concept and clothing product orientation by integrated design of businesses marketing. Although fashion display design is a small factor, the overall visual appearance style it reflects can directly affect the clothing marketing.

\section{Fashion Display Design Is the Communication Link Between Businesses and Consumers}

As an important link in the clothing products marketing, the role of fashion display design is specific and small. Fashion display design reduce the distance between consumes and products by creating some specific scenes and atmospheres. In these specific scenes and atmospheres, consumers can easily create the association related to their actual life, in the process of creating association, the distance between products and consumers is reduced, and the buying desire of consumers is stimulated. Because different consumers have different aesthetic standards and preferences, the fashion display design with different styles can meet their different requirements. With the assistance of fashion display design, consumers can choose their favorite products according to their preferences. In this process, fashion display design make a role of a bridge of communication between consumers and businesses. 


\section{Technical Application of Fashion Display Design}

So how to make fashion display design? And which aspects of innovation more attention should be paid to? Next, let us make a brief elaboration and discussion for these questions.

\section{Application of Display Design in Fashion Style Design}

In the premise of insisting on the clothing product style and inner qualities, fashion display design should pursue the practical and art mutual integration and innovation on the basis of the whole harmony and adopt different forms of display design to create the different appearance effect of clothing products in order to meet the diversified requirements of consumers on personalized clothing design and consumption. For example, in the design of window fashion display, clothing matching design should be applied to show rich and fashionable clothing culture by creating the whole clothing styles and clothing levels; soft, exquisite and smooth silk, appropriate leopard print with wildness and pierced silk stockings are adopted to make people feel not only the distinguished clothing style but also the fashionable breath.

\section{Application of Display Design in Space Layout}

\section{Clothes Shelve Design}

What is the core of a fashion store? There is no doubt that clothes shelve is the core of a fashion store. The clothes shelve has very important position in a fashion store, so it is very important to make good display design for clothes shelves. The implementation of its layout and sensory effect should begin with the general layout of clothes, at the same time, more attention should be paid to the deep design and multi-dimensional design of the overall display effect of clothes shelves. In addition, it should be noted that the clothes with similar styles should be displayed in the same area as far as possible. In short, the design effect of a clothes shelve is very important which can directly affect the buying desire of consumers.

\section{Design of Mannequin Model of Clothes}

In the fashion display design, the mannequin model is also a powerful tool used to increase effectiveness. It should be remembered that the display design of mannequin model must be not angular whether in fashion store or in the clothes window. An excellent display of mannequin model should be natural as an integration of the actual operation and the aesthetic artistry, and it can fully show its effectiveness. The space design of mannequin model should be carried out according to the pattern, style, color and material of clothes in order to bring the direct visual enjoyment to the consumers.

\section{Design of Wall and Floor}

In the display design, the design of wall and floor is easier to ignore which covers the biggest area. The effect brought by the design of wall and floor is also very critical and should not be ignored which can greatly affect the visual effect of consumers. The design of wall and floor should begin with some important aspects such as graphic design and decorative design in order to create a whole environmental effect and stimulate the buying desire of consumers. At the same time, a specific artistic style for ideal design effect of wall can be created with the assistance of some ornaments used to decorate wall and the effect of light.

\section{Application of Display Design in Color Design}

Color is the key of the whole design which can directly affect the responses of consumers with critical and direct effect. It is noteworthy that more attention should be paid to the impactive visual effect of color. The organization form of color, texture and other visual appearance effects are expressed according to the physiological function of color and other essential attributes to deliver the experience of aesthetic sensibilities and build artistic environment of color, then color design bring the contagion effect to the consumers to make them with the relaxed mood be in the atmosphere built by color design.

\section{Application of Display Design in Texture Design}

Here, the texture design mainly includes two aspects the design of clothes own and the design of the props related to the clothes display. However, many texture forms are formed by the relevant 
means according to the design concept to make products have more special inner appeal and integrate marketing with art for the best design effect.

\section{Conclusion}

In conclusion, under the background of rapid changes of times and the constant integration of various factors, clothing industry should be fully aware of the importance of clothes display design and make good application of clothes display design. Clothes display design is a bridge between products and consumers, the everlasting appeal and connotation of products can be directly showed to the consumers better to attract their attention and buying desire in order to increase the sales of products with the assistance of this bridge. On the basis of the full awareness of the importance of clothes display design of clothes industry, more innovation and application space should be gave to display design. The design connotation should be created and showed through some aspects such as color, pattern, matching and environment with the clothes design as the starting point to make clothing products be full of beauty and vitality in order to attract attention and buying desire of consumers. With the deepening of China's WTO entry, the clothing industry of our country recognizes its own many disparity and questions. The clothes display design developed late in our country, so there are many problems and faults for clothes display design. Enterprises and the relevant people should promote the clothes display design to develop to a higher level according to their own characteristics on the basis of fully studying foreign advanced level and experience. Only in this way can an excellent clothes display design be carried out, and the role of clothes display design be fully played.

\section{References}

[1] Ying Wen, Jianfeng Zhang. Discussion on Men's Clothing Display Design Elements of 2010[J]. Journal of Zhejiang Textile and Fashion College, 2011(01)

[2] Yi Zhong. "I am a craftsman of fashion but not a designer-Dutch Artist StefanieNieuwenhuyse"'[J]. China Garment, 2013(09)

[3] Color Marketing: Color Plus Pattern Is Equal to the Half of Purchase Rate. Pattern Design With Appropriate Popular Color in spring and summer of 2014[J]. China Garment, 2013(09).

[4] Susanna Lau. Selected Fashion Works of the Royal College of Art[J]. China Garment, 2013(09)

[5] Zi Wan. Fashion Boutique Club-Appreciation of Display of Boutique Retail of Germanic Fashion Brand Kaiser[J]. China Garment, 2013(09)

[6] Xiaoyu Wang. Research on Database Construction Based on Protection of the Han Costumes Pattern[J]. Journal of Zhengzhou University(Philosophy and Social Sciences), 2013(05)

[7] Xiaobo Li. Expression of Style of Window Display Design of Clothing Brand - With the Chinese-Style Clothing Brands as Examples[J]. MING(Attitude), 2017(02)

[8] Chunhai Zhang. Research on Display Design Based on Market Economy - Principle of Color Application[J]. China Market, 2016(31)

[9] Qiang Tang, Fangfang Xin. Curriculum Planning and Teaching Exploration of Fashion Display Design[J]. Art and Design(Theory), 2011(06)

[10] He Liu, Meilian Yu. Innovation of Modeling Forms Design of Clothing Store Display[J]. Journal of Nantong University: Social Science Edition, 2008(4). 